\title{
Analysis of composite fuel application possibility based on coal and oats husks in industrial power engineering
}

\author{
Stanislav Yankovsky ${ }^{1, *}$, Anton Tolokolnikov ${ }^{1}$, and Vladimir Zaytsev ${ }^{1}$ \\ ${ }^{1}$ National Research Tomsk Polytechnic University, 634050 Tomsk, Russia
}

\begin{abstract}
Experimental studies were conducted to determine the energy and technical characteristics of composite fuels from $\mathrm{T}$ grade coal (Alardinsky deposit) and oats (Shegarsky district, Tomsk region). An effective concentration of composite fuel of $50 \% / 50 \%$ is established, at which the heat of combustion is reduced by less than $2 \%$, the ash content is up to $44 \%$, and the fly ash output is up to $48 \%$.
\end{abstract}

\section{Introduction}

Efforts have been intensively conducted in many developed countries (China, India, the USA, Canada, Russia, etc.) over the last years to create scientific basis for so-called composite fuels application in power engineering: coal-water $[1,2]$ and organic coal-water slurry [3,4], fuel based on products of agricultural production [5,7], etc.

This trend of recent decades is due, in the main, to fairly objective criteria: uncertainty with the prospects of using gas and oil products in the next 15-20 years; solution of the tasks of sulfur, nitrogen and carbon oxides emissions reduction into the atmosphere; objective need to burn waste of many industrial production and agriculture. The simplest and, possibly, most productive solutions in practice are technologies of composite fuels application based on coal and biomass in power engineering.

It is known [5] that coal is the main energy resource for thermal power plants and boiler houses. Large expenses for fuel supply to remote areas of the Russian Federation are the main reason of rising prices for heat consumers [5]. Energy strategy of the Russian Federation foresees an increase in the share of coal in heat and electricity production in the fuel balance of the country, as well as development of renewable energy sources [5-7]. In recent years, the share of biomass in energy resources production has been increasing [7]. The most common bioresources are wood, wood waste and waste from agricultural production of cereals, annual accumulation of which is up to one hundred million tons. In recent years, the pace of agricultural development in the Russian Federation is growing rapidly, so it is possible that utilization of waste from this sector of production will take an important place in the future [7].

A common way of agricultural waste utilization is by burning. The most developed recycling technologies are in Bangladesh, Thailand, India, Indonesia, Malaysia, USA [7].

\footnotetext{
${ }^{*}$ Corresponding author: jankovsky @,tpu.ru
} 
Oats husks have low calorific value in comparison with coal, which negatively affects its promising application in thermal and electric energy production. But on the other hand, low ash content of this biofuel is attractive for solving the problem of power engineering negative impact reduction on the environment.

The result of mixing high-ash energy coal with oats husks can allow obtaining high synergetic effect. However, the use of such composite fuels in Russia is not developed due to the lack of both general theory of their component composition formation and combustion. Technical, energy and environmental characteristics of produced composite fuels, including addition of oats husks to coal, have not been sufficiently studied experimentally.

Urgency of the problem of agro-industrial complex wastes recycling with the use of composite fuels is confirmed by numerous publications (for example, [8-14]), which give results of fuels properties study based on biomass and coals during combustion in power plants. But results of [8-14] are not the basis for consistencies analysis of composite fuels thermal decomposition processes with a change in the ratio of biomass and coal concentration. Analysis of composite fuels creation possibility with energy characteristics close to the initial coal and significantly better environmental parameters (close to biofuels) is of great interest.

\section{Results of conducted experiments and discussion}

Technical and energy characteristics of composite fuels have been determined. Quantity of $\mathrm{T}$ grade coal (Alardinskoe deposit, particle size less than $80 \mu \mathrm{m}$ ) and oats husks (Shegarsky district of Tomsk region, particle size less than $200 \mu \mathrm{m}$ ) were loaded at controlled mass concentrations into galvanized drum of Pulverisette 6 planetary mill [8]. Mixing process was conducted at drum rotation speed of $500 \mathrm{rpm}$ for seven minutes. Technical analysis of composite fuels was carried out after mixing. Change in calorific value of composite fuels is shown in Figure 1.

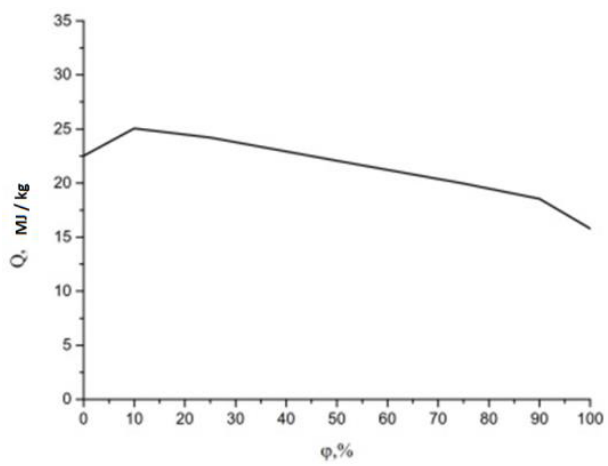

Fig 1. Effect of oats husks concentration on calorific value of composite fuel in relation to homogeneous coal ( ${ }^{\varphi}$ - oats husks share).

Analysis of the results shown in Fig. 1 describes that increase in oats husks concentration leads to synergistic effect. Calorific value of composite fuel relative to the initial coal with $10 \%$ biomass concentration increases by $11 \%$, with $25 \%$ concentration by $7 \%$, with oats husks content of $50 \%$ calorific value of the fuel is reduced only by $2 \%$.

Based on the foregoing, it can be concluded that an increase in oats husks concentration up to $25 \%$ leads to improvement in energy performance of composite fuels compared to coal. An increase of up to $50 \%$ reduces them by only $2 \%$. 
An important indicator of environmental impact during thermal decomposition of fuel is its ash content. Figure 2 shows dependence of change in fuel ash content obtained in experiments from increase of biomass concentration.

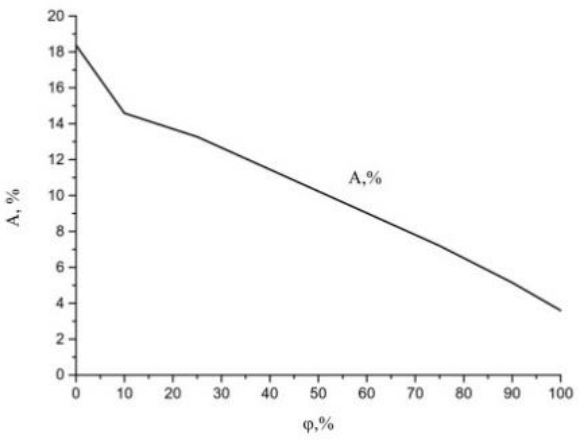

Fig 2. Effect of oats husks concentration on composite fuel ash content in relation to homogeneous coal ( ${ }^{\varphi}$ - oats husks share).

Figure 2 shows that increase in oats husks concentration in composite fuel leads to significant decrease in ash content. Thus, when concentration of biomass is increased to $50 \%$, ash content is reduced by $44 \%$. The obtained results allow drawing conclusion about positive influence of biomass on energy and ecological characteristics of composite fuel based on coal and oats husks.

The main and important parameter characterizing fuel is yield of volatile substances. Figure 3 shows experimentally established dependence of volatile substances release from oats husks concentration in composite fuel (relatively to homogeneous coal).

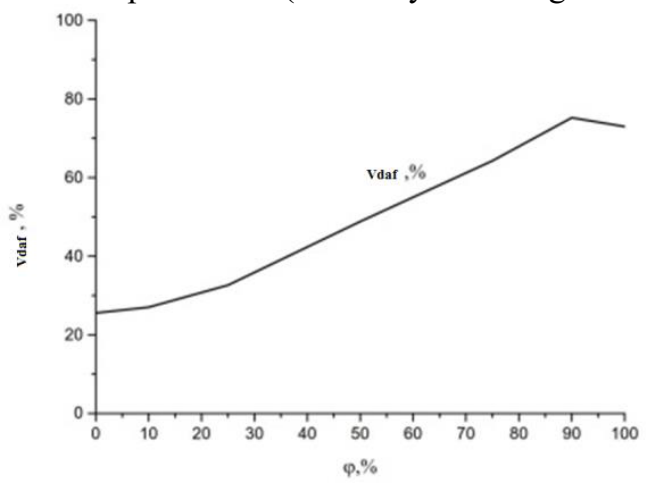

Fig 3. Effect of oats husks concentration on composite fuel volatiles yield in relation to homogeneous coal ( ${ }^{\varphi}$ - oats husks share).

Analysis of $\operatorname{Vdaf}\left({ }^{\varphi}\right)$ dependence shows an increase in volatile substances yield with increase of biomass concentration in composite fuel. Thus, when husks concentration in composite fuel is $50 \%$, volatiles yield is $90 \%$.

\section{Conclusion}

Influence of oats husks concentration on technical and energy characteristics was established based on the results of conducted experiments, as well as parameters of volatiles release during thermal decomposition of composite fuel on the basis of coal and biomass. 
Results of carried out experimental research allow drawing conclusion that composite fuel based on coal and oats husk can be used for combustion in furnaces of steam and water-heating boilers.

The research has been accomplished with financial support of the Russian Federation Ministry of Education and Science within implementation of the project No 13.7644.2017/BCH.

\section{References:}

1. S.V. Syrodoy, G.V. Kuznetsov, V.V. Salomatov, Therm. Eng., 62, 10 (2015)

2. G.V. Kuznetsov, V.V. Salomatov, S.V. Syrodoy, Combustion, Explosion and Shock Waves, 51, 4, (2015)

3. K.Y. Vershinina, D.O. Glushkov, G.V. Kuznetsov, P.A. Strizhak, Solid Fuel Chem. 50, 88 (2016)

4. K. Y. Vershinina, J. Eng. Thermophys. 90, 1 (2017)

5. M. Jumoke, Applied Energy 188, 215 (2017)

6. A.V. Fedyukhin, I.L. Maikov, V.A. Sinelshchikov, Book of Abstracts of International Conference on Interaction of Intense Energy Fluxes with Matter (2011)

7. W. Yan, S. Wang, D.G. Zhou, C. Xing, Y. Zhang, G.M. Pharr, Wood and fiber science 44, 1 (2009)

8. K.V. Slyusarskiy, K.B. Larionov, V.I. Osipov, S.A. Yankovsky, V.E. Gubin, A.A. Gromov, Fuel, 191, 383 (2017)

9. N. David, Z. John Cofiring Biomass and Coal for Fossil Fuel Reduction and Other Benefits-Status of North American Facilities in 2010 (USDA, Washington DC, 2010)

10. M. Van der Stelt, H Gerhauser, J. Kiel, K. Ptasinski, Biomass Bioenergy, 35, (2011)

11. L. Nunes, J. Matias, J. Catalo, Renew Sustain Energy Rev. 40, (2014)

12. K. Larionov, I. Korjashov, S. Jankovskij, V. Gubin, A. Zenkov, MATEC Web Conf. 37, (2015)

13. K.B. Larionov, I.V. Mishakov, A.A. Gromov, A.V. Zenkov, MATEC Web Conf. 91, (2016)

14. D.V. Gvozdyakov, D.V. Gvozdyakov, V.E. Gubin, Chem. Petr. Eng. 51, (2015) 